\title{
Diseños de investigación y pruebas estadísticas utilizadas en revistas odontológicas de la red SciELO
}

\section{Research Designs and Statistical Tests Used in Dental Journals of the SciELO Network}

Navarro $\mathrm{P}^{*}$, Chuhuaicura $\mathrm{P}^{* *}$, Soto-Faúndez $\mathrm{N}^{* * *}$, Soto $\mathrm{C} * *$.

\section{RESUMEN}

Introducción: Los principios y metodologías de la medicina basada en evidencia, constituye un importante campo de atención, mediante el cual se incorpora, de manera reflexiva toda la información disponible, con la finalidad de brindar al paciente las mejores opciones para los problemas de salud de la población. Conocer el tipo de evidencia y los análisis estadísticos utilizados mejoran las aptitudes del clínico para tomar cualquier decisión. El objetivo de este estudio fue identificar los diseños de investigación y pruebas estadísticas utilizadas en revistas de odontología indexadas en la red SciELO entre el año 2000 y 2016.

Material y método: Se realizó un estudio bibliométrico descriptivo de corte transversal. La búsqueda incluyó todas las revistas del área odontológica indexadas en la base SciELO (Scientific Electronic Library Online), entre los años 2000 y 2016, con disponibilidad en idioma español. Se excluyeron revistas que no presentaran información online disponible, sin acceso o que fueran eliminadas de la base de datos. La búsqueda se realizó desde el portal web de SciELO (http://www.scielo.org/php/index.php), a fin de obtener acceso a toda la red, a través de la sección "colecciones de revistas", donde se encontraron los links de acceso para cada país.

Resultados: Se analizaron 634 artículos desde el primer semestre del 2000 al segundo semestre del 2016. Los Diseños de estudio más utilizados fueron el estudio corte transversal ${ }^{(244)}$ y el estudio in vitro ${ }^{(107)}$. De las pruebas estadísticas paramétricas ANOVA ${ }^{(121)}$ y t-test para muestras independientes ${ }^{\left({ }^{(9)}\right.}$ fueron las más frecuentemente utilizadas y de las pruebas no paramétricas, fue Chi cuadrado de Pearson ${ }^{(113)}$ y U-Mann Whitney ${ }^{(70)}$.

Discusión: Las diferencias observadas entre los diferentes diseños de estudios se mantiene similar a lo que se había reportado anteriormente, siendo diseño de corte transversal el más utilizado. Comprender e interpretar los de métodos estadísticos permitirán identificar resultados relevantes y aplicables para la toma de decisión clínica.

PALABRAS ClAVE: Study design, Statistical Analysis, Dentistry, SciELO.

Departamento de Odontología Integral Adultos, Facultad de Odontología, Universidad de La Frontera, Temuco, Chile.

Universidad Autónoma de Chile, Chile.

Centro de Investigación en Ciencias Odontológicas (CICO), Facultad de Odontología, Universidad de

La Frontera, Temuco, Chile.

*** Escuela de Odontología, Facultad de Odontología, Universidad de La Frontera, Temuco, Chile. 


\section{SUMMARY}

IIntroduction: The principles and methodologies of evidence-based medicine constitute an important field of attention, by means of which all the available information is incorporated in a reflexive manner, in order to provide the patient with the best options for health problems. The population. Knowing the type of evidence and the statistical analyzes used improve the clinician's abilities to make any decision. The objective of this study was to identify the research designs and statistical tests used in dentistry journals indexed in the SciELO network between 2000 and 2016.

Material and method: A cross-sectional descriptive bibliometric study was carried out. The search included all the dental journals indexed in the SciELO database (Scientific Electronic Library Online), between 2000 and 2016, with availability in Spanish. We excluded journals that did not present online information available, without access or that were eliminated from the database. The search was made from the SciELO web portal (http://www.scielo.org/php/index.php), in order to obtain access to the entire network, through the section "magazine collections", where the access links for each country were found.

Results: 634 articles were analyzed from the first semester of 2000 to the second semester of 2016 . The study designs most used were the cross section study ${ }^{(244)}$ and the in vitro study ${ }^{(107)}$. Of the parametric statistical tests $\mathrm{ANOVA}^{(121)}$ and t-test for independent samples ${ }^{(96)}$ were the most frequently used and non-parametric tests, it was Pearson's Chi Square ${ }^{(113)}$ and U-Mann Whitney ${ }^{(70)}$.

Discussion: The differences observed between the different study designs remains similar to what had been previously reported, with the cross-sectional design being the most used. Understanding and interpreting the statistical methods will allow to identify relevant and applicable results for clinical decision making.

KEY WORDS: Study design, Statistical Analysis, Dentistry, SciELO.

Fecha de recepción: 16 de noviembre de 2018.

Fecha de aceptación: 28 de noviembre de 2018.

Navarro P, Chuhuaicura P, Soto-Faúndez N, Soto C. Diseños de investigación y pruebas estadísticas utilizadas en revistas odontológicas de la red SciELO. 2019; 35, (1): 19-25.

\section{INTRODUCCIÓN}

Actualmente estamos inmersos en una época de gran desarrollo tecnológico, donde el ejercicio de la práctica clínica ha cambiado su forma de diagnosticar y tratar enfermedades. Durante los últimos años el concepto de Odontología Basada en Evidencia (OBE) ha ido cobrando cada vez más relevancia definiéndose como un proceso sistemático en el cual el profesional realiza la toma de decisiones en base a la mejor información biomédica posible, con la finalidad de entregar al paciente las mejores opciones disponibles para dar solución al problema de salud que presente ${ }^{(1)}$.

La utilización de un enfoque basado en evidencia puede ayudar a los clínicos que quieren mantenerse al día con los cambios en sus áreas de trabajo, asistiéndolos en la selección de artículos relevantes, en la extracción y aplicación de esta información, así como también, orientándolos en la toma de decisiones en el ámbito de la Salud Pública, buscando lograr un equilibrio entre los criterios de equidad, eficiencia y calidad en la resolución de problemas de salud ${ }^{(2,3)}$.

La evidencia se clasifica de acuerdo al enfoque epidemiológico clínico, considerando para tal efecto el diseño de investigación que lo originó (4). Los diseños se pueden clasificar de acuerdo a la manipulación de la variable de estudio, si existe una intervención artificial de la variable el estudio es experimental ${ }^{(5)}$. Por otro parte, en los estudios observacionales se debe especificar la temporalidad en la que se obtendrá la información, si se realiza en un solo momento el diseño del estudio será transversal. Mientras que un estudio longitudinal requerirá obtener la información a través del seguimiento de una cohorte de 
individuos determinada ${ }^{(6)}$. Por último, si el objetivo del estudio es reportar las características de una población determinada el diseño de investigación es descriptivo, asimismo los diseños de investigación analíticos generalmente buscan identificar la relación causal entre las variables.

Los estudios experimentales son aquellos que se caracterizan porque su metodología lleva implícita la intervención, es decir, se valoriza el efecto de una o más intervenciones, y el carácter prospectivo está dado por la recolección de la información y el seguimiento de la población en estudio. Frente a esto se considera que la fuente primaria de más evidencia científica los ensayos clínicos controlados y randomizados que permiten comparar la efectividad de diferentes intervenciones terapéuticas ${ }^{(7)}$.

Finalmente es necesario que el profesional clínico pueda identificar los diferentes diseños de investigación y la validez de estos estudios en términos metodológicos (análisis crítico) determinando si pueden ser transferible a la práctica clínica y la realidad local. Esto permitirá escoger el diseño más adecuado enfocándose principalmente en discriminar qué tan distintos pueden llegar a ser los pacientes del estudio respecto de aquellos a quienes se pretende aplicar la información ${ }^{(8)}$.

El objetivo de esta investigación fue establecer mediante análisis bibliométrico, los diseños de estudios y pruebas estadísticas utilizadas en revistas de odontología indexadas en la red SciELO entre el año 2000 y 2016.

\section{MATERIAL Y MÉTODOS}

Se realizó un estudio bibliométrico descriptivo de corte transversal, en el cual se incluyeron todas las revistas del área odontológica indexadas en la base SciELO (Scientific Electronic Library Online) en idioma español, portugués e inglés, desde el primer semestre del año 2000 al segundo semestre del año 2016. Se seleccionaron artículos originales y de revisión. Aquellas revistas que no contaran con acceso online disponible o que no presentaran información en relación a la base de datos consultada fueron excluidas del estudio. En el portal web SciELO se realizó la búsqueda utilizando la metodología propuesta por Cantín \& Aravena a través del en- lace "colecciones de revistas", que permite obtener acceso a los sitios SciELO de cada país y, por lo tanto, a sus colecciones ${ }^{(9)}$.

Una vez en el sitio SciELO de cada país, la búsqueda se realizó a través del buscador "lista por materia", luego se continuó con el enlace "ciencias de la salud", el que entregó los títulos de las revistas relacionadas con el área "odontología". De esta manera, los artículos para el estudio se seleccionaron a través de un muestreo no probabilístico por conveniencia.

Los datos recopilados fueron ingresados en tablas de doble entrada donde la información obtenida fue analizada mediante estadística descriptiva.

\section{RESULTADOS}

De acuerdo a los resultados obtenidos, Se identificaron 16 revistas odontológicas indexadas en SciELO. El país que presentó la mayor cantidad fue España con 3 revistas, a continuación, Chile y Colombia con 2 revistas cada uno. Se analizaron 634 artículos desde el primer semestre del 2000 al segundo semestre del 2016, la mayor cantidad de estudios analizados correspondió a la Revista clínica de Periodoncia, Implantología y Rehabilitación Oral (Chile) ${ }^{(93)}$, International Journal of Odontostomatology (Chile) ${ }^{(85)}$, Revista Odontológica Mexicana (México) ${ }^{(82)}$, Avances en Odontoestomatología (España) ${ }^{(65)}$, Revista Cubana de Estomatología (Cuba) ${ }^{(52)}$ (Figura 1)

En relación a los diseños de estudio se puede destacar que la mayor frecuencia la presentaron los estudios de corte transversal (244), estudios in vitro $^{(107)}$, reportes de un caso ${ }^{(71)}$, Revisión de la literatura $^{(41)}$, Estudios de cohorte ${ }^{(39)}$ y ensayos clínicos aleatorizados ${ }^{(35)}$. De forma aislada se observaron estudios como Experimento ex vivo, series de casos, Experimentos en animales, revisiones sistemáticas. (Figura.2)

Por otro parte, se observó que las revistas que utilizaron más pruebas estadísticas en sus artículos fueron, Revista Clínica de Periodoncia, Implantología y Rehabilitación Oral(153), International Journal of Odontostomatology ${ }^{(150)}$, Revista Facultad de Odontología Universidad de Antioquia(100), Revista de Odontología da UNESP(75), (Figura 3) también se obtuvo que 194 artículos utilizaron el análisis 


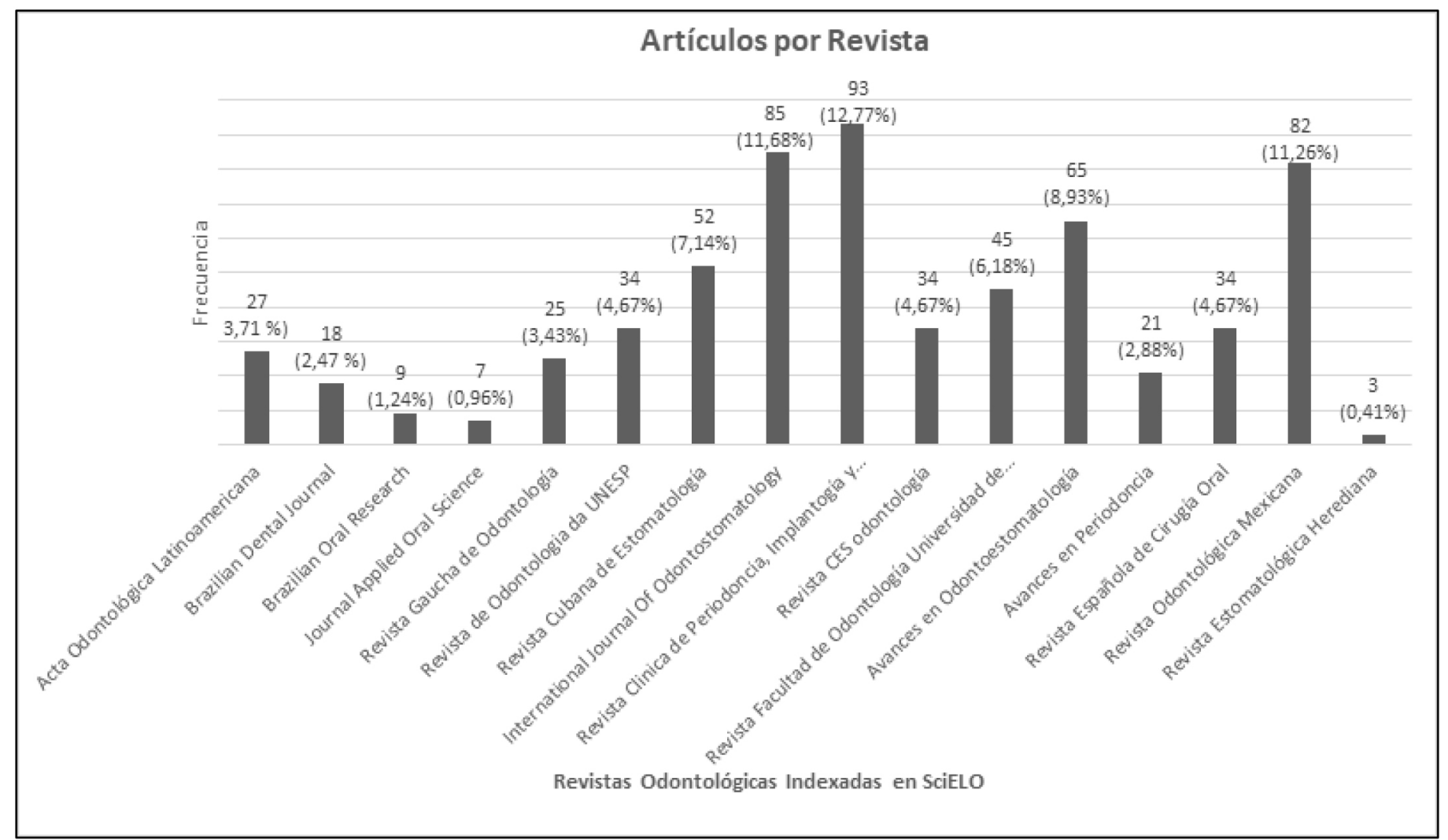

Figura 1. Distribución de frecuencias de artículos por revista indexada en la red SciELO.

descriptivo como base para el posterior análisis inferencial. Mientras que las pruebas estadísticas paramétricas más utilizadas fueron ANOVA $^{(121)}$ y t-test para muestras independientes (96) y de las pruebas no paramétricas, Chi cuadrado de Pearson $^{(113)}$ y U-Mann Whitney ${ }^{(70)}$. De las pruebas aplicadas el $12,9 \%$ corresponde a ANOVA, que a su vez representa el 49,7\% de las pruebas paramétricas. En relación a las pruebas no paramétricas, Chi- cuadrado de Pearson corresponde al 12,1\% de las pruebas aplicadas. (Figura 4)

\section{DISCUSIÓN}

En relación al diseño de investigación más utilizado en las revistas odontológicas de la red SciELO, fue el corte transversal, este diseño se caracteriza por evaluar de forma simultánea la exposición y la enfermedad de una población definida, en un periodo determinado ${ }^{(6)}$ su objetivo principal es describir frecuencia y distribución de una característica y debido a su naturaleza descriptiva, estos diseños no pueden establecer causalidad ${ }^{(10)}$. Los resultados de este estudio concuerdan con los datos obtenidos en la investigación previamente realizada por Navarro et al. Este diseño presenta ventajas, ya que algunos autores mencionan que son relativamente económicos y más rápidos en cuanto a su ejecución ${ }^{(11)}$, así también dentro de sus desventajas, está la situación temporal, ya que el estudio se desarrolla solo en una instancia por lo que el contexto podría proporcionar resultados diferentes si se hubiese elegido otro marco de tiempo, además como se mencionó anteriormente no se puede establecer relación causal ${ }^{(12,13)}$.

Continuando con los diseños de investigación, el segundo más frecuentemente aplicado, corresponde a los estudios in vitro, este resultado se contrasta a lo reportado por Navarro et al. 2016 donde el segundo lugar correspondió a los estudios de casos y controles. En cuanto al estudio in vitro estos son realizados en dispositivos de laboratorio utilizando tejidos, células o moléculas, donde se intenta simular las condiciones naturales en que se realizó la observación ${ }^{(14)}$. En el área odontológica, es de esperar encontrar estudios in vitro, ya que, durante las últimas décadas, este diseño de investigación ha permitido evaluar la eficacia 


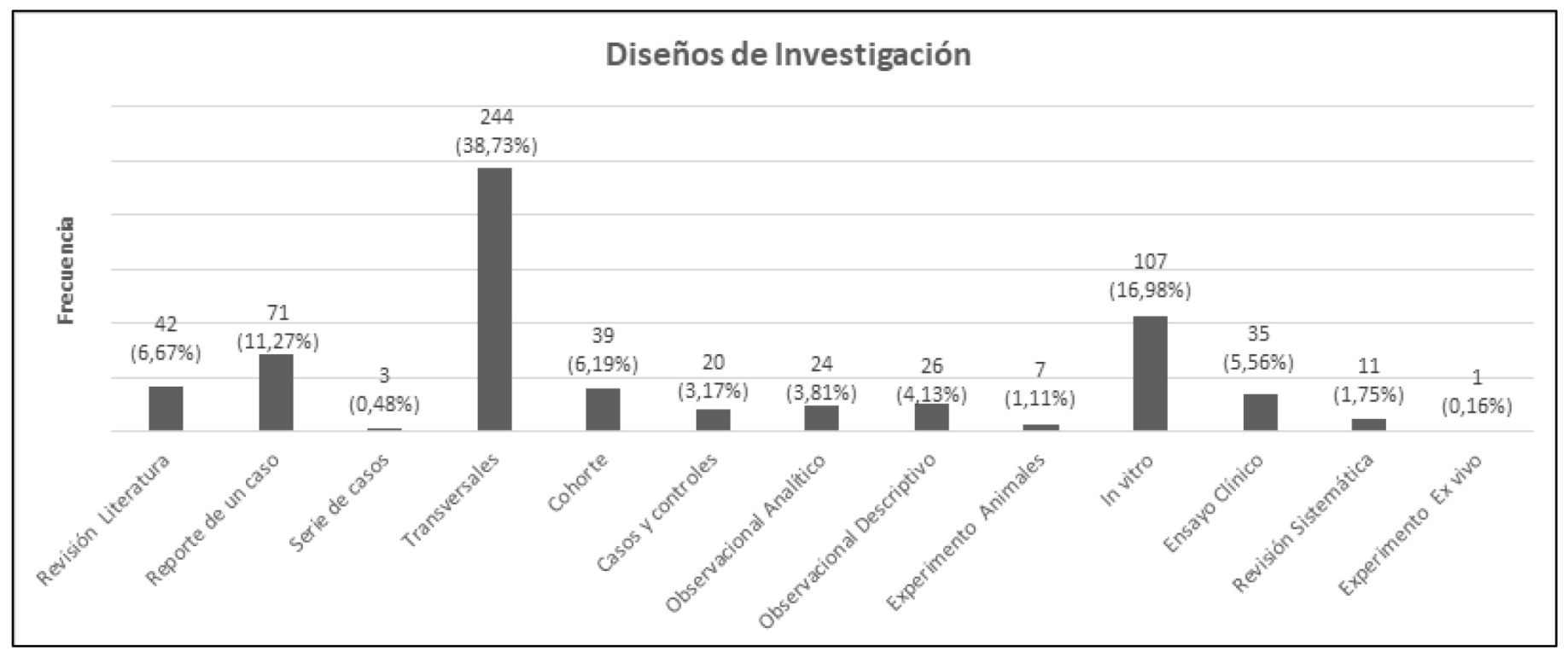

Figura 2. Distribución de frecuencias de diseños de investigación utilizados en las revistas odontológicas de la red SciELO.

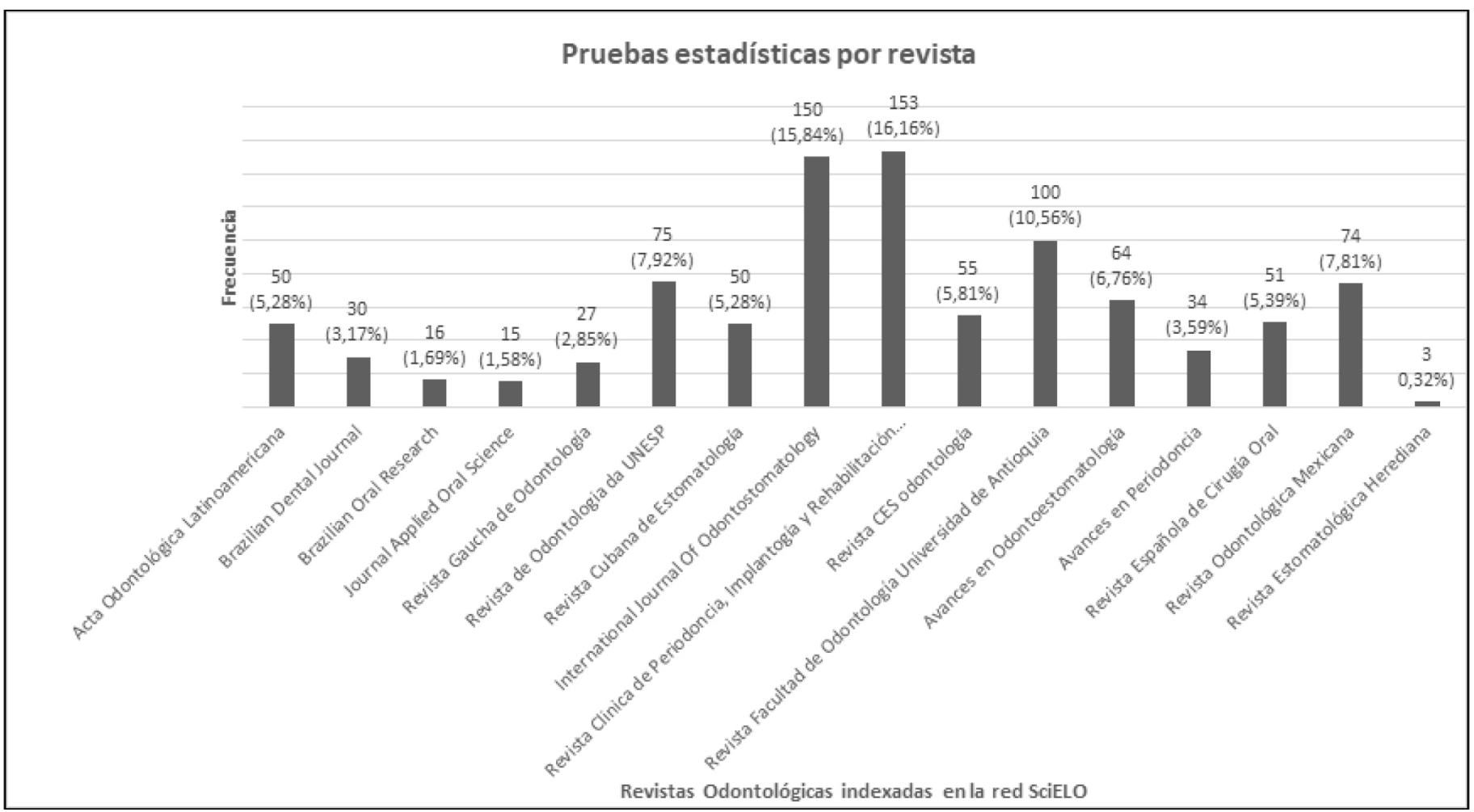

Figura 3. Distribución de frecuencias de pruebas estadísticas por revista odontológica indexada en SciELO.

de diferentes biomateriales, así como también la implementación de nuevas técnicas para mejorar la calidad de la práctica clínica. Sin embargo, en cuanto a la calidad de la información los diseños de estudios encontrados en mayor proporción no entregan evidencia suficiente para tomar decisiones clínicas basadas en la evidencia ${ }^{(5,15)}$.
De acuerdo a las pruebas estadísticas, estas aportan herramientas que van desde el análisis e interpretación de datos hasta el proceso de predicción y toma de decisiones, sin embargo, es necesario que los investigadores tengan conocimientos básicos y que puedan aplicar la estadística sin llegar a resultados equivocados ${ }^{(16)}$. Las 


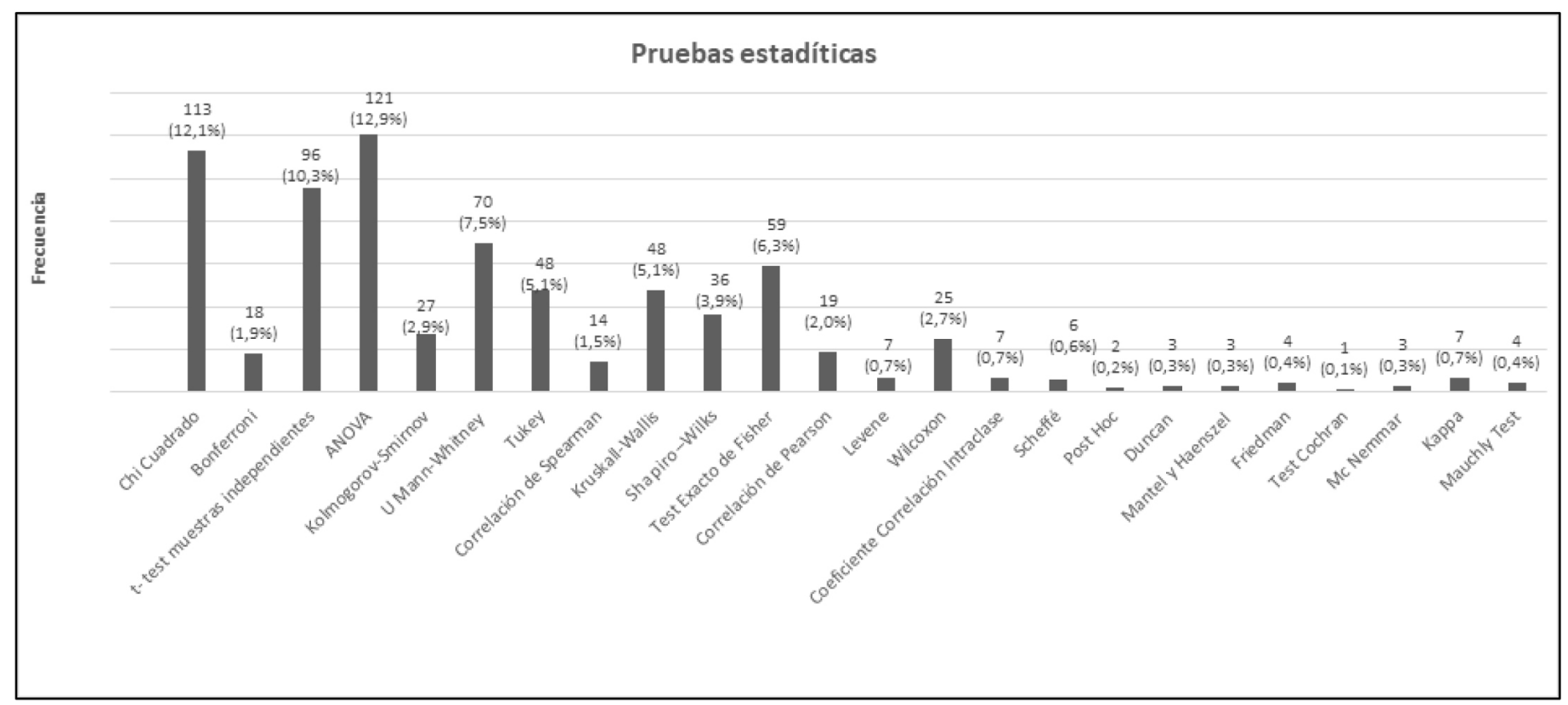

Figura 4. Distribución de frecuencias de pruebas estadísticas paramétricas y no paramétricas más utilizadas en los artículos publicados en revistas odontológica SciELO.

aplicaciones de la estadística permiten identificar diferentes métodos para analizar los resultados en los artículos de las revistas odontológicas indexadas en la red SciELO, donde se estimó una mayor proporción de estudios que emplearon estadística inferencial versus solo estadística descriptiva, además dentro de las pruebas utilizadas, la descrita más frecuentemente fueron las pruebas paramétricas, entre ellas ANOVA y T-test para muestra independiente. La prueba de ANOVA o análisis de varianza prueba la hipótesis de que las medias de más de dos poblaciones son iguales, mientras que $\mathrm{T}$ - test se diseñó para examinar las diferencias entre dos muestras independientes que tengan distribución normal y homogeneidad en sus varianzas ${ }^{(17)}$.

Más allá de conocer el significado de cada prueba, es de gran importancia que tanto el investigador como el lector, identifique y comprenda el resultado que arrojan estas, ya que estudios publicados anteriormente concluyen que existe un escaso conocimiento para interpretar resultados de investigaciones clínicas ${ }^{(18,19)}$.

\section{CONCLUSIONES}

Las diferencias observadas entre los diferentes diseños de estudios se mantienen similar a lo que se había reportado anteriormente, sin embargo, en el área odontológica, se encontró que los estudios in vitro, cada vez se realizan más especialmente por las temáticas que tratan. En relación a las pruebas estadísticas, las pruebas paramétricas son las más utilizadas en el contexto de los análisis inferenciales, además se concluye que saber interpretar los resultados de cada prueba facilitará la comprensión de los estudios y su posterior aplicación clínica.

\section{BIBLIOGRAFIA}

1. Carrasco Labra, A., Brignardello Petersen, R. (2008). Odontología Basada en Evidencia, Revista Dental de Chile, 99 (2), pp. 32-37.

2. Beltrán, O. (2003) Redefinición de la medicina basada en evidencias. Revista Colombiana de Gastroenterolología, 18, pp. 102- 106.

3. Ballini, A., Capodiferro, S., Toia, M., Cantore, S., Favia, G., De Frenza, G. et al. (2007). Ebvidence-based dentistry: what's new? International Journal of Medical Sciences,4, pp. 174- 178.

4. Díaz-Narváez VP, Calzadilla-Núñez A. Artículos científicos, tipos de investigación y productividad científica en las ciencias de la sa- 
lud. Rev Cienc Salud. 2016;14(1): 115-121.

5. Navarro, P., Cantín, M., Ottone, NE. (2016). Diseños de investigación utilizados en revistas odontológicas de la red SciELO: Una visión bibliométrica. Avances en Odontoestomatología, 32(3), pp.153-158.

6. Vallejo, M. (2002). El diseño de investigación: una breve revisión metodológica. Archivos de Cardiología de México,.72, pp. 8-12.

7. Doig, G. S. E Simpson, F. (2005). Randomization and allocation concealment: a practical guide for researchers. J. Crit. Care, 20(2) pp.187-91.

8. Sutherland, S. (2001). Evidence-based dentistry: part V, Critical appraisal of the dental literature: papers about therapy. Journal Canadian Dental Association, 67. pp. 442- 445.

9. Cantín, M. and Aravena, Y. (2014). Las Revistas Odontológicas en la Base SciELO: Una Mirada Bibliométrica. International Journal of Odontostomatology, 8(2), pp. 215-220.

10. García de la Torre, G and Huerta-Alvarado SG. (1998). Consideraciones metodológicas y análisis simple de los estudios transversales. Bol Med Hosp Infant Mex; 55(6): 348-356.

11. Levin, K. (2006). Study design III: Cross-sectional studies. Evidence-Based Dentistry, 7(1), pp.24-25.

12) Mann, C. (2003). Observational research methods. Research design II: cohort, cross sectional, and case-control studies. Emergency Medicine Journal, 20(1), pp.54-60.

13. García Marcos, L., Guillén Pérez, J., and Orejas
Rodríguez-Arango, G. (1999). Epidemiología y metodología aplicada a la pediatría (V): Sesgos, Anales españoles de pediatría,50, pp. 519.552

14) Fina, BL., Lombarte, M., and Rigalli, A. (2013). Investigación de un fenómeno natural: ¿Estudios in vivo, in vitro o in silico?, Actualizaciones en osteología, 9(3), pp.239-240.

15. Hernández-Ávila, M., Garrido-Latorre, F., López-Moreno, S. (2000) Diseños de estudios epidemiológicos. Salud Pública de México, 42(2), pp. 144-154.

16. Navarro, P., Ottone, NE., Acevedo, C., Cantín, M., (2016b) Pruebas estadísticas utilizadas en revistas odontológicas de la red SciELO, Avances en Odontoestomatología, 33(1), pp. 25-32.

17) Flores-Ruiz, E., Miranda-Novales, MG., Villasís-Keever, MÁ (2017). El protocolo de investigación VI: cómo elegir la prueba estadística adecuada. Estadística inferencial. Revista Alergia México, 64(3), pp. 364-370

18) Windish, D., Huot, S. and Green, M. (2007). Medicine Residents' Understanding of the Biostatistics and Results in the Medical Literature. JAMA, 298(9), p.1010.

19) Susarla, S. and Redett, R. (2014). Plastic Surgery Residents' Attitudes and Understanding of Biostatistics: A Pilot Study. Journal of Surgical Education, 71(4), pp.574-579.

\section{AUTOR DE CORRESPONDENCIA:}

Dr. Pablo Navarro Cáceres

Centro de Investigación en Ciencias Odontológicas Facultad de Odontología, Universidad de La Frontera Av. Francisco Salazar 01145, Temuco, Chile E-mail: pablo.navarro@ufrontera.cl

Tel.: +56 2596646 